\title{
Perspectives of 3D printing technology in orthopaedic surgery
}

\author{
Zamborsky $\mathrm{R}^{1}$, Kilian $\mathrm{M}^{2}$, Jacko $\mathrm{P}^{3}$, Bernadic $\mathrm{M}^{4}$, Hudak $\mathrm{R}^{5}$ \\ Department of Orthopedics, Faculty of Medicine, Comenius University and National Institute \\ of Children's Diseases, Bratislava, Slovakia. mirokilian@seznam.cz
}

\begin{abstract}
Using most widespread technology of rapid prototyping (RP) in medicine focus on the development of models for diagnosis, for training and planned surgery, as well as the direct manufacture of implants for bone reconstruction. The applications of 3D printing in the field of medicine are giving extraordinary results and tissue and prosthetic 3D printing, medical and engineering research professionals are conducting 3D printing organ bind. Researchers worldwide are pursuing the creation of artificial bone using 3D printers, bones that can be later implanted to humans. In near future, many body parts could be manufactured in a turn and successfully implanted to patients. Although medical advances in 3D printing are used in orthopaedic field, research in 4D printing has already started. Flat objects made with 3D printing, using a regular plastic, combined with smart material, were able to become a hub without an external intervention. In nutshell, the future of additive manufacturing (AM) in trauma and orthopedic surgery is relatively bright with the inclusion of 3D printing in medicine. Bioprinting in this area will be focused on fractures, nonunions, deformities and bone, cartilage and soft tissue reconstruction. CONCLUSION: The innovative technology not only assists the medical staff but is also beneficial for the patients because the medical problems, which were not curable in the past, are now possible with modern technology (Fig. 4, Ref. 52). Text in PDF www.elis.sk.

KEY WORDS: bone defect, tissue engineering, 3D printing, biomaterials, bone, porous scaffold.
\end{abstract}

\section{Introduction}

The 3D printing technology in medicine is becoming fashionable peripherals that nobody doubts, but their uses of time they surprise us every day, too. Not so long ago, plans to manufacture a functional weapon with a 3D printer were spread across internet. Fortunately, not all is going to be that dangerous kinds of applications. The University of Victoria in Wellington (New Zealand) is developing a new generation of splints for broken bones that have

${ }^{1}$ Department of Orthopedics, Faculty of Medicine, Comenius University and National Institute of Children's Diseases, Bratislava, Slovakia, ${ }^{2} 2$ nd Orthopedic and Traumatology Department, University Hospital Saint Cyril and Metod and Comenius University, Bratislava, Slovakia, ${ }^{3}$ Department of Traumatology, Slovak Medical University and University Hospital, Bratislava, Slovakia, ${ }^{4}$ Department of Traumatology and Orthopedics, University Hospital, Trnava, Slovakia, and ${ }^{5}$ Department of Biomedical Engineering and Measurement, Faculty of Mechanical Engineering, Technical University of Kosice, Kosice, Slovakia

Address for correspondence: M. Kilian, MD, 2nd Orthopedic and Traumatology Department, University Hospital Saint Cyril and Metod and Comenius University, Antolska 11, SK-85107 Bratislava, Slovakia.

Acknowledgement: This work was supported by Slovak Research and Development Agency Project: "Manufacturing and testing of custom made bone scaffolds made of hydroxyapatite (HA) by use of 3D printing technology”, APVV-14- 0294, Research of additive manufacturing of biodegradable magnesium alloys and their applications in implantology and regenerative medicine APVV-17-0278, Analysis of PEEK polymer and possibilities of its additive manufacturing for medical applications, APVV-15-0356 and project VEGA, 1/0179/19 Research, development and testing of bioreactor for cultivation of tissues and organs after bioadditive manufacturing. called Cortex. The system is based on a combination of technologies ranging from $\mathrm{X}$-ray radiographs with the three-dimensional scan of the patient. Once all data are combined, they produce a model printed on a 3D printer. The benefits of this system are several, lower weight versus a traditional plaster, the possibility of a higher density of clamping where the injury requires, and the breathability of the cast itself as it is not the classic system plaster, skin suffers much less and prevents injury therein (1). That is just an example of the new era of producing models, implants, scaffolds or even living organ tissue. Rapid prototyping (RP), additive manufacturing (AM) or 3D printing are just a synonymous of modern technology and related research rapidly penetrating into a medical field.

Therefore, to provide the readers a proper understanding of the future of additive manufacturing in trauma and orthopedic surgery, we have highlighted the different aspects of 3D printing in medicine. To provide further information on this area, different specific segments are discussed in the paper. For instance, we have identified the significance of 3D medical printing with rapid prototyping, similarly, preoperative model printing in fractures. In this regard, there are many areas that are associated with 3D printing in orthopaedics and trauma.

\section{Rapid prototyping technologies in medicine focusing on surgery}

Ciocca, et al (2) highlighted that the advances in new rapid manufacturing technologies immediately came to medicine and 
in particular had a major impact on reconstructive surgery of tissues and organs mainly by the combination of three phenomena:

- Advances in computing and the explosion in knowledge, development and application of imaging techniques (CT, USS, and MRI).

- Techniques of CAD / CAM can be reproduced with micron accuracy regarding the form, dimensions and morphology of tissues and organs to repair or replace.

- Several of the tested materials and these technologies (RMT) have proven biocompatibility (2).

Of all applications of these techniques, those related to biomedical engineering occupy third place with $15 \%$. They could be applied in areas ranging from training, planning and procedures in surgery to manufacture prostheses and implants, both to reconstruct soft tissue (arteries, muscles, tendons, etc.) and for the reconstruction of bone tissue and the manufacture of various types of implants. In this sense, it is clear that both are useful in manufacturing somato-prosthesis (reconstructing external anatomy defects) as well as different types of prostheses and implantable devices. Using most widespread technology of rapid prototyping in medicine focus on the development of models for diagnosis, for training and planned surgery, as well as the direct manufacture of implants for bone reconstruction. All of which represent almost $60 \%$ of applications. Approximately $14 \%$ of investigations relating to the development of prototypes for tissue engineering, which currently appears as the field of application more important, is associated with $40 \%$ of scientific articles published in the last five years on the use of rapid prototyping in bioengineering (3). In Biomedical Engineering, specialties that are most applicable, are radiology, medical imaging, anatomy, surgery, orthopedics and dentistry, which occupy $50 \%$ of the total.

\section{Osteochondral reconstruction}

The availability of new procedures based on evidence is fundamental to the advancement in the treatment of cartilage injuries, the most valuable asset in our joints. Minimally invasive methods, such as arthroscopy, will play a decisive role in the future in this field. The technique that allows the regeneration of hyaline cartilage with all its properties, arthroscopic osteochondral autologous transplantation, is presented as a simple, economical solution that requires only one surgical time and is therefore provided at cost and aggressiveness to solve the problem of focal lesions of the cartilage, as it allows a hyaline cartilage transplant fully formed with its subchondral bone (4). It has been proved that the vitality of the transplanted tissue and the technique is associated with a high percentage of short-term success by providing pain relief and good joint function. However, monitoring is essential to have a longer time period and new evaluations, both clinical outcome and its biomechanical function, so you can definitely unseat the "traditional techniques".

The impossibility of hyaline cartilage to repair their own injuries makes the treatment of focal articular cartilage loss a topic of constant debate in our field and the treatment is not solved yet.
Classically, surgeons have to use techniques that stimulate the bone marrow and only get the formation of fibrocartilage tissue, well below the normal hyaline cartilage mechanical properties. Recently, the revolutionary advent of transplantation techniques cartilage cells has opened a new and promising way to rebuild damaged hyaline cartilage, but it still should be considered an experimental technique, to prove their long term validity (5). Controversy remains regarding the cell type of choice, bone marrow stromal cells or chondrocytes $(6,7,8,9,10,11)$.The focus in osteochondral reconstruction remains in provision of microenvironment to facilitate cell migration and differentiation. Many authors employ a combination of cells, growth factors and scaffolds and in this concept, the two options are available - scaffold only (12), and cell only techniques (13). The majority of meniscal injuries require a suture or removal of the torn meniscus. Long term clinical studies on the results of avascular zone meniscal repair are sparse (14). 3D printed porous polycarbonate-urethane (PCU) and ultra-highmolecular-weight polyethylene (UHMWPE) blend for the artificial meniscus and future perspectives were already published (15).

\section{Preoperative model printing in fractures and patient specific surgical guides}

3D printing also shows a great impact in this area, mostly in manufacturing dimensionally accurate human anatomy models from the high resolution image data. The digital image could be obtained from either computer tomography or magnetic resonance imaging (16). The data can be exported in common medical file format DICOM (digital imaging and communication in medicine). After the segmentation and rendering, the data could be converted to stereolithography format (STL) and printed as 3D virtual model. It could be a bone deformity, malunion, bone tumor or complex intraarticular fractures $(17,18)$. This method is used mostly in preoperative surgical planning and explaining the complex surgical operations to the resident doctors and patients (Fig. 1).

In the orthopaedic oncology, it may also be a valuable tool in virtual diagnostic and pre-surgical planning $(19,20)$. The models can be made in any color which you can distinguish between different tissues (vessels, or pathology from the healthy tissue) and such modes are used for teaching purposes in various hospitals worldwide. Using some special preoperative planning software, we are now able to print out custom made osteosynthetic material (e.g. screws, plates) out of titanium (21, 22, 23, 24).

Also, the research is ongoing in creating computer designs involved in the production of soft and rigid polymers that serve as manufacturing material for a $3 \mathrm{D}$ printer. The results so far obtained offer a copy that is up to 22 times more resistant to break that the one present in a patient. In the article by Professor Markus Buehler, this indicates that the printed bone is strong and resilient and consists of its two constituent materials, soft collagen protein and hydroxyapatite mineral rigid. Researchers at MIT have developed a system, which prints 3D synthetic bones using a 3D printer in materials combining two synthetic polymers that have a similar bone structure behavior. The patterns used as materials include some natural and nacre although some are synthetic compounds. 

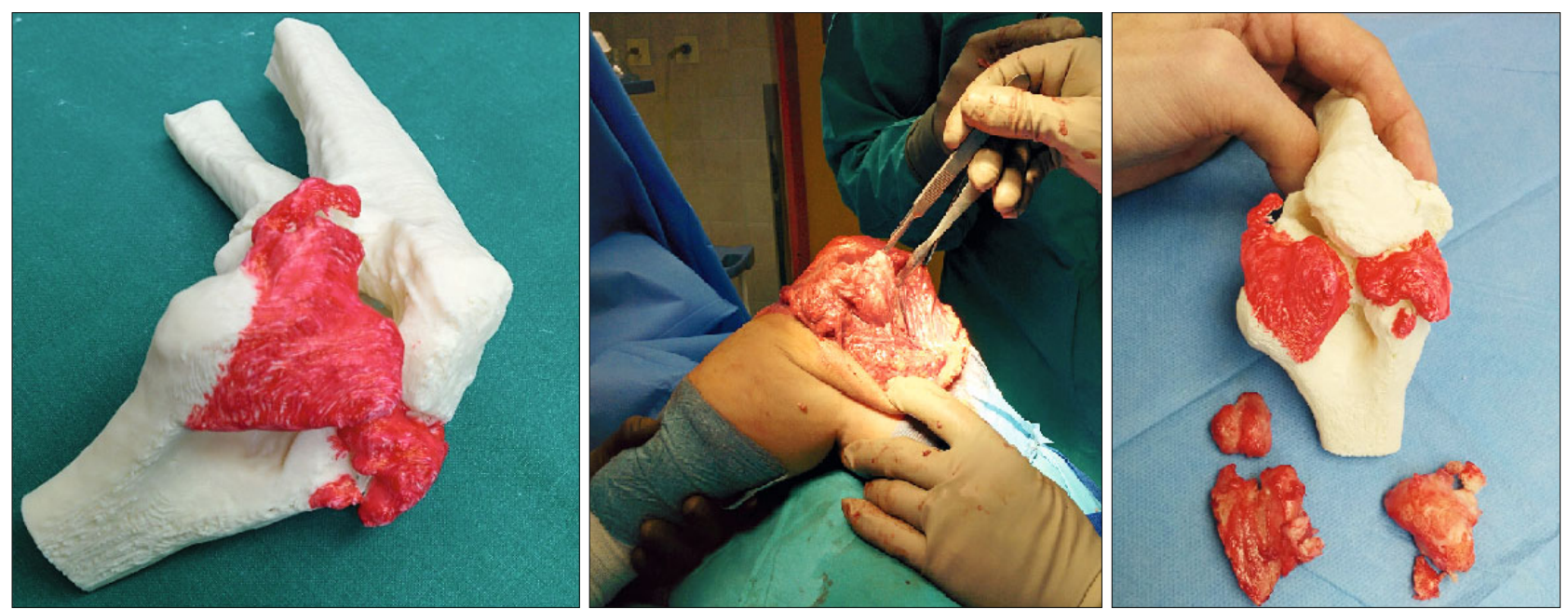

Fig. 1. Real size printed three-dimensional anatomic template for virtual preoperative planning of elbow surgery (Department of Trauma surgery, UniversityHospital in Bratislava).

After obtaining the first results, the researchers responsible for this project have tested 3D printed bones for their strength and endurance to stress and extreme mobility. The conclusion from these tests is that the $3 \mathrm{D}$ printed bone shows a higher resistance to fracture, up to 22 times above normal bone, and is able to better withstand the natural condition.

With Professor Buehler, students Leon Dimas and Graham Bratzel have collaborated on this research declaring their satisfaction with making a model capable of replacing natural bone in case the patient needs it (25). As for the application of 3D printing in general, they said that designing their own models with an improved quality could improve the existing products even today.

\section{Custom orthopedic implants, instruments and plastic or met- al implant prototypes}

Currently, medical implants demand to be better adapted to the patient. Thus, the concept of 3D printing is growing in expectation and is common to hear news about important clinical advances achieved due to this new technological reality. At this point, it is important to note that within the concept of 3D printing a lot of manufacturing technologies are framed by addition (wire deposition, laser sintering, electron beam melting, etc.), as well as different materials for manufacturing (plastics like ABS or nylon, metal, etc.). In particular, in the world of orthopedic and maxillofacial surgery, where traditionally metallic materials (stainless steel, alloys of cobalt chrome or titanium) have been used, there are additive manufacturing technologies, such as melt electron beam (EBM) or selective laser melting (SLM), which are being involved in the realization of the custom implants because they are able to work with such material with a high quality (26).

Thanks to the flexibility that this technology can bring to the production with respect to the traditional techniques of production, such as the machining of parts from forging or casting, a number of important advantages can be introduced to the final establish- ment of these technologies. We already can create many geometric designs that we're unable to do with other existing and traditional technologies, like hip cups or full part total knee prosthesis. AM would reduce a part's cost, compared to traditional manufacturing techniques, up to 80-90\%. The aim is to develop products that are either highly complex, highly customized or where the quantity needed is small and other production techniques are cost-effective. This also includs manufactured drilling and cutting guides and other metal and plastic implants, that could be used in fixing the fractures. In addition, customizing implants allow clinics and hospitals to reduce the amount of products that must be stored since they don't have to encompass the full range of sizes. However, currently manufacturing traditional series does not allow the move towards personalization (27). Thus, one of the main objectives of the project was to allow implant manufacturers to offer these customized implants maintaining reasonable unit costs without the loss of quality, while lowering their manufacturing volumes. The future implant design is moving into porous or porous/solid implant constructs, which are already printable and AM has the unique ability to provide “complexity for a low price” (Fig. 2).

The biggest limitations will be in the mindset of the education of the individual involved in the process and the biocompatibility (28).

\section{Printing bone constructs - bone grafts}

It's been a while until the idea of 3D printing became popular in the world of manufacturing, which has been shown to provide major cost reductions throughout the manufacturing sector. At the desktop level, 3D printing is a method for any ordinary person to create prototypes of their ideas. To be able to print the bone, we need to consider a structure that is to some extent both flexible and strong, contains collagenous matrix components, and is favorable to mineralization and vascularization (29). It is also necessary to maintain the porosity and the ability to match the 

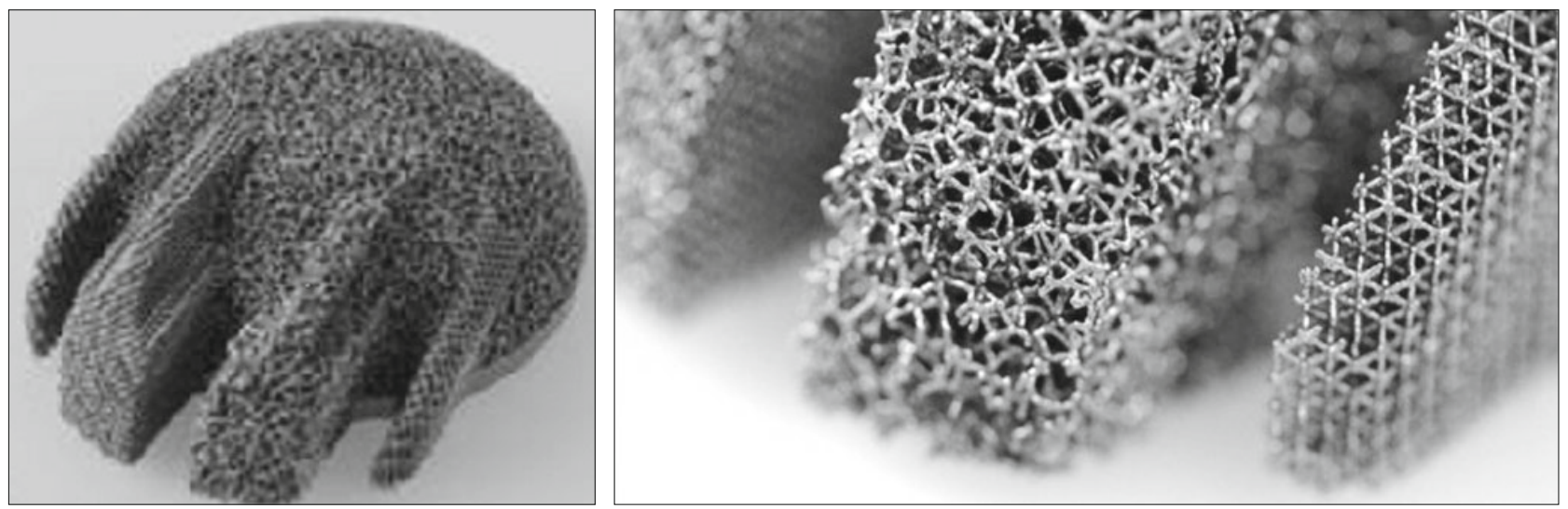

Fig. 2. 3D-printed poroustitaniumalloy (acetabular part) with bony ingrowthpotential.

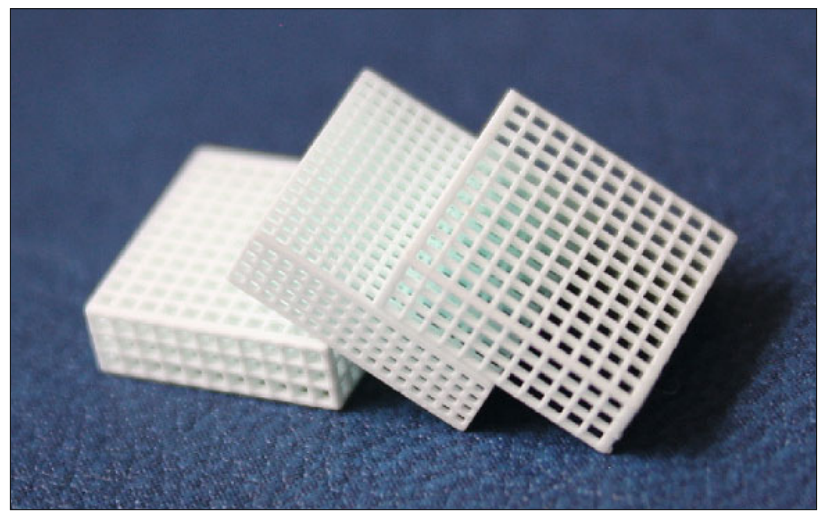

Fig. 3. Synthetichydroxyapatite (HA) 3D printedscaffoldwithcontrolledporosityfor bone replacement (Department of BiomedicalEngineering and Measurement, Faculty of MechanicalEngineering, TechnicalUniversity of Košice).

graft to the defect site. Traditional methods for the manufacture of synthetic bone graft, such as solvent casting/salt leaching, phase separation and foaming also have many limitations, such as shape restrictions, inconsistency and inflexibility and lack of control of porosity and pore interconnectivity, which are crucial in bone regeneration (30).

Reichert et al (31), successfully used rapid prototyping to produce bone graft material capable of mediating reconstruction of large bone defects, using the medical-grade PCL-TCP. These cylindric scaffolds with combination with BMP-7 or stem cells (bone marrow) could be a promising equivalent of bone healing compared to autologous bone graft.

Bio-impression 3D is the next phase in this evolution, as we have begun to see in the initial building blocks for a future, in which humans will one day be able to have an entirely new body 3D printed. This year, the company based in the Netherlands called Xilloc has taken a step forward in the field of bio printing, to reach an agreement with the Japanese company called Next 21 3D printing for bone in hospitals in Europe. This technology is intended to replace the current methods of graft including bone marrow transplant to other parts of the body, allogenic bone marrow transplanta- tion, and even the creation of bone substitutes hydroxiapatite and calcium phosphate (29). All these current technologies are slow in processes, which are not whole part bones, but only a small portions of artificial bone stock that can be implanted in the body and then grow slowly by itself (Fig. 3).

Next technology is known as CT 21-Bone, where the exact size and shape required by the bone is determined. Then using the $3 \mathrm{D}$ printing process ink, it prints actual 3D bone in the exact form required, including those suitable for inclusion of bone conduction and blood vessels areas. Xilloc begin manufacturing and selling CT Bone in several European countries, which allows doctors to begin implementation of technology in their patients. Unlike previous methods of bone graft, using heat, CT Bone does not. This allows the merger to take effect more quickly, and because the bone structure is printed, healing times are significantly improved. 3D printers are capable of printing with an accuracy of up to $0.1 \mathrm{~mm}$ (32). The results so far have not only shown to improve healing time, but also to help prevent infections and body rejections, which are considered a breakthrough in the field of medicine.

The current limitations are not only the biocompatibility and biodegradability issues, but also a stem cells and growth factors compatibility currently limited by the application of thermal or chemical treatment (33).

\section{Periprosthetic infection}

Periprosthetic of periimplant infection around the joint or in to the medullary canal is a devastating complication affecting about $1-3 \%$ of joint replacements and represent a huge problem in healthcare cost and is associated with a substantial patient morbidity $(33,34)$.

Pulido et al signified that the infection of a hip or knee is a feared complication that could spoil the success of joint replacement (35). The prosthetic surface germs can invade the neighboring bone (periprosthetic infection) and soft with an eventual formation of a fistula. Bloodstream invasion is followed by a toxic sepsis and until a few years ago it was a real tragedy not only for the joint function but also for the patient's life. Most of the first postoperative year infections are caused by exogenous 
germs from the patient's skin, contaminated particles entering by air, or surgical field. In some cases, the source of infection comes from a distant focus (throat, teeth, bladder, and urinary tract infection). Prosthetic infections can be classified as acute or early, chronic, blood-borne and those detected in suspected mechanical loosening. The diagnosis is based on puncture for bacteriological and pathological studies $(34,36)$. More than 25 $\%$ of revisions are attributed to these infections, which are expected to increase (35).

Surgical treatment may be by careful tactical and technical criteria appropriate to the particular case. Currently, the prognosis may be improved by some advanced technologies, although it depends on two key factors: Firstly, an early diagnosis of the infection, avoidance of prescribing antibiotics without a thorough prior bacteriological investigation to identify the seed and determine its sensitivity using the corresponding susceptibility $(36,37)$. And secondly - to consider an early implant removal and the use the antibiotic impregnated cement or a spacer and after 6-8 weeks to consider a reimplantation (38). Cemented spacer are most preferably made intraopeatively and are characterized by their fragility and suboptimal dimensions (39, 40, 41).

Rapid prototyping can help to solve this costly and devastating problem of the two-stage revision process for the implant associated infection by putting a cutting edge technology in producing selection of spacers. These technologies could also be focused on producing special antibiotic coated patient-specific spacers to perfectly fill a bone defect, vastly improving the biomechanical and biological conditions for new bone development and infection management of new bone development $(42,43,44)$.

\section{Spinal fusion}

The concept of spinal fusion is the common surgical procedure known as the surgery to enduringly connect two or more than two vertebrae in the spine by eliminating the motion between them (45). The supplementary tissue of the bone is either from patient - as autograft or is from the donor known as allograft and the spinal fusion particularly immobilizes parts of spine as it changes the motion of the spine. This places an extra strain and stress on vertebrae below and above the fused part. This can also increase the rate at which the area of the spine mainly degenerate. The process of fusing the spine (spinal arthrodesis) is mainly focused to eliminate the pain, which is caused by the abnormal movement of vertebrae by stopping faulty vertebrae, which is primarily caused by the degenerative conditions, deformity or trauma and the fusion mainly involves the techniques, which are designed to mimic normal healing. This treatment is also widely used to treat the patients who suffer from the thoracic and the cervical problems. There have already been an ongoing research in the use of ceramics in combination with the bone marrow aspirate (46). In selected cases, the cages may be used in combination with bone grafts or they may also be produced as titanium 3D printed products supported by osteoinductive and osteoconductive material with the combination of stem cells and growth factors. Prof. Dr. Uwe Spetzger, at the Department of Neurosurgery of the Klinikum

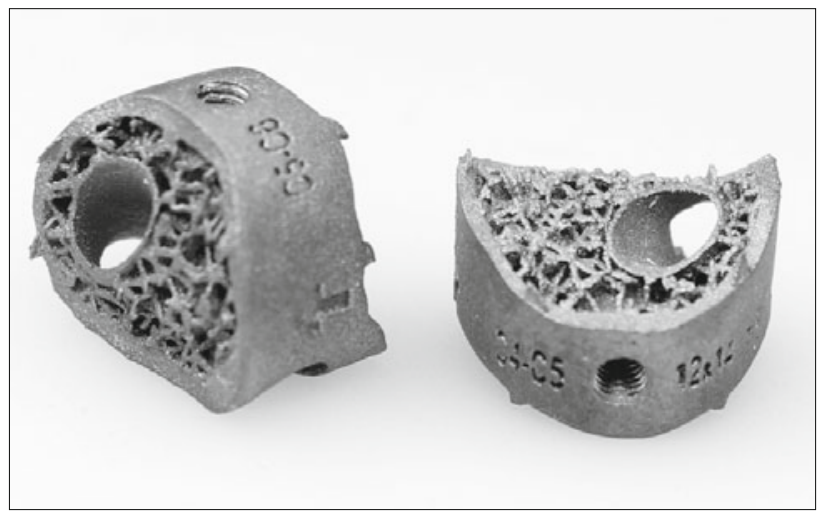

Fig. 4. 3D-printed prototype of individual porous spinal cages (CEIT Biomedical Engineering s.r.o., Košice).

Karlsruhe in Germany was responsible for the planning and execution of the new treatment, where the 3D printed titanium cage was designed in conjunction with 3D Systems Company and implanted to the patient. Such titanium implants has up to 80 percent porosity and essentially provides "scaffolding” ideal for growing bone cells and vascular structures. The implant was designed and built to mimic trabecular bone structure and specially fabricated for the individual patient. In practice, it means the implant is far less likely to shift or destroy the structural integrity of surrounding bone. Researchers say the method also prevents delays in the process of fusion. Fabrication of solid biodegradable materials with cell-laden hydrogels, would be the next step in evolution as a titanium or artificial bone filaments $(47,48)$. In Slovakia, there is also an ongoing research with individualized porous spinal cages designed by Biomedical Engineering, s.r.o. company and produced by DMLS 3D printing technology (Fig. 4).

\section{Challenges, problems and future trends in clinical use}

3D printing has advanced beyond the stage of testing in mice ear implant. Some of the human body tissues are able to be printed, with functionality implemented and working. Science, called regenerative medicine, has already succeeded in engineering of skin, cartilage, bladders, urine tubes and blood vessels and soon can be successfully implanted in patients (49). The structures need to be able to get the oxygen and nutrients to the tissues around until they developed their own blood vessels to supply. The Santo Grail of regenerative medicine has always been to be able to design complex organs such as the kidney, liver and heart. The organs are very dense and need the ability to leverage their own oxygen supply to ensure survival. Research has already been used to design rodents and pigs' heart, liver and lung. When repopulated with organ specific cells, organs have been able to produce some of the functions of the original organs in the laboratory. They are used in research due to similarities to humans when it comes to the structure and size of the organs. Anthony Atala demonstrates an early-stage experiment that could someday solve the problem of organ donors: A 3D printer that uses living cells to output a 
transplantable kidney (3). Using similar technology, Dr. Atala’s young patient Luke Masella, received a bladder designed 11 years ago; we find him on stage. Despite a huge progress in regenerative medicine and rapid prototyping there are still some key challenges unsolved. Vascularisation/creation of functional vascular network comprised of endothelial cells surrounded by a muscle layer seems to be the aim of research and testing. The other challenges are the cell source and growth factors. Longterm cellular viability is unpredictable, especially in combination with celltoxic scaffolds. There are still many limitations in the availability and commercialization of rapid prototyping/3D printing systems. The price of bioprinters and materials approved for medical use is still very high, therefore it is limited only for certain research groups and institutions.

Although medical advances in 3D printing are happening almost every day, there's more that can be done with 3D printed materials to make them more flexible and more useful. Structures that can transform in a pre-programmed way in response to a stimulus. Recently given the popular science name of "4D printing”, perhaps a better way to think about it is that the object transforms over time. The aim of the $4 \mathrm{D}$ printing is to make objects of a 3D printer to be able to reconfigure themselves into useful forms without outside assistance. Flat objects made with 3D printing, using regular plastic, combined with smart material, were able to become a hub without an external intervention (50). Despite how recently 4D printing has been introduced to the public, there are already several industries clamoring to be the first to test the many possibilities of this new technology. Medical field shouldn't stay far behind and in the future, the 4D printing can be things, such as:

- 4D Printing of Blood Vessels and Tissue (stents, artificial blood vessels and other replacements that respond to blood pressure, physical stress or blood flow in order to minimize blood loss or damage to surrounding tissues)

- Intelligent Bandages (4D materials that instinctively respond to the damaged tissue, feeding oxygen to the burn site to hasten healing and reduce the risk of infection. They could also respond to infection by producing antibiotics or other medications to short-circuit the spread of the infection. These "smart bandages" could dramatically reduce healing times for severe burn patients).

- Faster Wound Healing (with 4D printed latticework, however, not only could new skin grow over and around the lattice network, the lattice itself could respond to the trauma at the wound site and alter itself into the best possible form for seamless integration).

Scientists, researchers and medical specialists all agree that there are amazing possibilities from artificial limbs to repairing or replacing damaged tissues or even organs that can be produced with responsive bioactive materials $(51,52)$.

\section{Conclusion}

The future of additive manufacturing in trauma and orthopedic surgery is relatively bright with the inclusion of 3D printing in medicine, or possible 4D printing products in the distant future. The innovative technology not only assists the medical staff, but is also beneficial for the patients because the medical problems, which were not curable in the past are now possible with modern technology. Therefore, now it's the responsibility of the medical staff and the technology manufacturers to use innovative technology of 3D printing in everyday practice, strengthen the research to improve the availability and price. Nevertheless, Rapid Prototyping is not going to fully replace the traditional manufacturing methods, it can help to reduce lead time and tooling costs and can be exactly custom made. New bone scaffolds printed in a low temperature in a high resolution, that will be sufficiently strong, allowing the fluid exchange and cellular influx during the bone healing, will give us a great promise in advanced bone healing and replacement.

\section{References}

1. Curodeau A, Sachs E, Caldarise S. Design and fabrication of cast orthopedic implants with freeform surface textures from 3-D printed ceramic shell. J Biomed Mater Res 2000; 53 (5): 525-535.

2. Ciocca L, De Crescenzio F, Fantini M, Scotti R. CAD/CAM and rapid prototyped scaffold construction for bone regenerative medicine and surgical transfer of virtual planning: a pilot study. Comput Med Imag Graph 2009; 33 (1): 58-62.

3. Peltola SM, Melchels FP, Grijpma DW, Kellomäki M. A review of rapid prototyping techniques for tissue engineering purposes. Ann Med 2008; 40 (4): 268-280.

4. Magill $\mathbf{P}$ et al. Osteochondral reconstruction and grafting. J Orthop Surg 2011; 19 (1): 93-98.

5. Wiewiorski M et al. Autologous matrix-induced chondrogenesis aided reconstruction of a large focal osteochondral lesion of the talus. Arch Orthop Trauma Surg 2011; 131 (3): 293-296.

6. Araki $\mathbf{S}$ et al. Improved quality of cartilage repair by bone marrow mesenchymal stem cells for treatment of an osteochondral defect in a cynomolgus macaque model. Acta Orthop 2015; 86 (1): 119-126.

7. Berninger MT et al. Treatment of osteochondral defects in the rabbit's knee joint by implantation of allogeneic mesenchymal stem cells in fibrin clots. J Visual Exp 2013; 75: e4423.

8. Koerner $\mathbf{J}$ et al. Equine peripheral blood derived progenitors in comparison to bone marrow-derived mesenchymal stem cells. Stem cells 2006; 24 (6): 1613-1619.

9. Reyes $\mathbf{R}$ et al. Comparative, osteochondral defect repair: stem cells versus chondrocytes versus bone morphogenetic protein-2, solely or in combination. Eur Cell Mater 2013; (25): 351-365.

10. Medvedeva $\mathbf{E}$ et al. Repair of damaged articular cartilage: Current approaches and future directions. Internat J Mol Sci 2018; 19 (8): 2366.

11. Daly AC et al. 3D printed microchannel networks to direct vascularisation during endochondral bone repair. Biomaterials 2018; 162: 34-46.

12. Castro NJ, Hacking SA, Zhang LG. Recent progress in interfacial tissue engineering approaches for osteochondral defects. Ann Biomed Engin 2012; 40 (8): 1628-1640.

13. Kon $\mathbf{E}$ et al. A novel nano-composite multi-layered biomaterial for treatment of osteochondral lesions: technique note and an early stability pilot clinical trial. Injury 2010; 41 (7): 693-701. 
14. Bostan B et al. Effects of different bone marrow stimulation techniques on avascular zone meniscal defects. Bratisl Med J 2018; 119 (10): 630-635.

15. Borges RA, Choudhury DZ. Min. 3D printed PCU/UHMWPE polymeric blend for artificial knee meniscus. Tribol Internat 2018; 122: 1-7.

16. Olejnik $P$ et al. First printed $3 D$ heart model based on cardiac magnetic resonance imaging data in Slovakia. Bratisl Med J 2018; 119 (12): 781-784.

17. McGurk M et al. Rapid prototyping techniques for anatomical modelling in medicine. Ann Royal Coll Surg Engl 1997; 79 (3): 169.

18. Brouwers $\mathbf{L}$ et al. The Value of 3D Printed Models in Understanding Acetabular Fractures. 3D Print Addit Manufact 2018; 5 (1): 37-46.

19. Gregory TM et al. Three-dimensional (3D) printing technology for the classification of complex distal humerus fractures. Ann Joint 2018; 3.

20. Hennessy DW, Anderson ME, Lozano-Calderón SA et al. Complex pelvic reconstruction using patient-specific instrumentation and a 3D-printed custom implant following tumor resection. J Hip Surg 2018; 2 (2): 61-66

21. Chung KJ et al. Preshaping plates for minimally invasive fixation of calcaneal fractures using a real-size 3D-printed model as a preoperative and intraoperative tool. Foot Ankle Internat 2014; 35 (11): 1231-1236.

22. Jeong $\mathbf{H}$ et al. Minimally invasive plate osteosynthesis using 3D printing for shaft fractures of clavicles. Arch Orthop Trauma Surg 2014; 134 (11): 1551-1555.

23. Rankin TM et al. Three-dimensional printing surgical instruments: are we there yet? J Surg Res 2014; 189 (2): 193-197.

24. Upex P, Jouffroy P, Riouallon G. Application of $3 D$ printing for treating fractures of both columns of the acetabulum: benefit of pre-contouring plates on the mirrored healthy pelvis. Orthop Traumatol Surg Res 2017; 103 (3): 331-334

25. Esses SJ et al. Clinical applications of physical 3D models derived from MDCT data and created by rapid prototyping. Amer J Roentgenol 2011; 196 (6): W683-W688.

26. Zdravkovič $\mathbf{M}$ et al. A case of using the Semantic Interoperability Framework for custom orthopedic implants manufacturing. Ann Rev Control 2012; 36 (2): 318-326.

27. Graichen $\mathbf{F}$ et al. Implantable 9-channel telemetry system for in vivo load measurements with orthopedic implants. IEEE Transact Biomed Engin 2007; 54 (2): 253-261.

28. El-Hajje A et al. Physical and mechanical characterisation of 3Dprinted porous titanium for biomedical applications. J Material Sci Material Med 2014; 25 (11): 2471-2480.

29. Fedorovich NE et al. Organ printing: the future of bone regeneration? Trends Biotechnol 2011; 29 (12): 601-606.

30. Kühne JH et al. Bone formation in coralline hydroxyapatite: effects of pore size studied in rabbits. Acta Orthop Scand 1994; 65 (3): $246-252$.

31. Reichert JC et al. A tissue engineering solution for segmental defect regeneration in load-bearing long bones. Sci Translat Med 2012; 4 (141): 141ra93-141ra93.

32. Öhman D et al. Mandible reconstruction with patient-specific implants: Case report of five consecutive patients. Internat J Oral Maxillofac Implants 2019; 34 (1).
33. Meseguer-Olmo L et al. In-vivo behavior of Si-hydroxyapatite/polycaprolactone/DMB scaffolds fabricated by 3D printing. J Biomed Material Res Part A 2013; 101 (7): 2038-2048.

34. Zimmerli W, Trampuz A, Ochsner PE. Prosthetic-joint infections. New Engl J Med 2004; 351 (16): 1645-1654.

35. Pulido $\mathbf{L}$ et al. Periprosthetic joint infection: the incidence, timing, and predisposing factors. Clin Orthop Rel Res 2008; 466 (7): 1710-1715.

36. Parvizi J et al. New definition for periprosthetic joint infection: from the Workgroup of the Musculoskeletal Infection Society. Clin Orthop Rel Res 2011; 469 (11): 2992.

37. Kapadia BH, Berg RA, Daley JA, Fritz J, Bhave A, Mont MA. Periprosthetic joint infection. Lancet 2016; 23 (387): 386-394.

38. Zajonz D et al. Diagnostic work-up strategy for periprosthetic joint infections after total hip and knee arthroplasty: a 12-year experience on 320 consecutive cases. Patient Safety Surg 2015; 9 (1): 20.

39. Buttaro MA et al. Intraoperative synovial C-reactive protein is as useful as frozen section to detect periprosthetic hip infection. Clin Orthop Rel Res 2015; 473 (12): 3876-3881.

40. Classen $\mathbf{L}$ et al. Two-stage revision total knee arthroplasty in cases of periprosthetic joint infection: an analysis of 50 cases. Open Orthop J 2015; 99): 49.

41. Dairaku K et al. Antibiotics-impregnated cement spacers in the first step of two-stage revision for infected totally replaced hip joints: report of ten trial cases. J Orthop Sci 2009; 14 (6): 704-710.

42. Berend KR et al. Molded Articulating Cement Spacers for Two-Stage Treatment of Infected THA and TKA. Surg Techn Internat 2015; (26): 343-349.

43. Pfitzner $T$ et al. A novel technique for a stable intramedullary fixation of static spacers in two-stage septic revision TKA with severe bone defects. Z Orthop Unfallchir 2015; 153 (2): 203-205.

44. Cicuéndez M et al. Multifunctional $\mathrm{pH}$ sensitive $3 \mathrm{D}$ scaffolds for treatment and prevention of bone infection. Acta Biomaterial 2018; 65: 450-461

45. Ritz U et al. A New Bone Substitute Developed from 3D-Prints of. Novel Biomaterial Tissue Engin 2018: 2019 (18): 360.

46. Rajaee SS et al. Spinal fusion in the United States: analysis of trends from 1998 to 2008. Spine 2012; 37 (1): 67-76.

47. Burleson J, Dipaola Ch et al. 3D Printing in Spine Surgery. In: 3D Printing in Orthopaedic Surgery. Elsevier, 2019; 105-122.

48. Fischer ChR et al. A systematic review of comparative studies on bone graft alternatives for common spine fusion procedures. Eur Spine J 2013; 22 (6): 1423-1435.

49. Bertjiaume F, Maguire TJ, Yarmush ML. Tissue engineering and regenerative medicine: history, progress, and challenges. Ann Rev Chem Biomol Engin 2011; (2): 403-430.

50. Mironov $\mathbf{V}$ et al. Organ printing: tissue spheroids as building blocks. Biomaterials 2009; 30 (12): 2164-2174.

51. Akbari $S$ et al. 4D Printing and Its Biomedical Applications. 3D and 4D Printing in Biomedical Applications: Proc Engin Addit Manufactur 2019; 343-372.

52. Gao F et al. Direct 3D printing of high strength biohybrid gradient hydrogel scaffolds for efficient repair of osteochondral defect. Adv Funct Material 2018; 28 (13): 1706644. 